\title{
El rol de la estructura familiar en el acortamiento de la estatura (baja talla por edad) de preescolares argentinos entre dos a cinco años
}

\author{
Beatriz Novak y María Eugenia Muniagurria \\ El Colegio de México, México/ \\ University of Wisconsin-Madison, Estados Unidos
}

Resumen

El presente estudio investiga la relación entre estructura familiar y acortamiento de la estatura en preescolares argentinos (dos-cinco años). Los datos utilizados corresponden a la primera ronda de la Encuesta Nacional de Nutrición y Salud 2005 ( $\mathrm{N}=11$ 632). Aunque las características socioeconómicas tienen un efecto significativo sobre el acortamiento, nuestro análisis identifica el rol independiente que tiene la estructura familiar. Los resultados de las regresiones logísticas muestran que las chances de acortamiento de preescolares en familias nucleares extensas son menores que las de aquellos en familias estrictamente nucleares. El pertenecer a una familia monoparental no aumenta las chances de acortamiento con respecto a las de un niño en una familia con ambos padres. Nuestro trabajo ofrece evidencias sobre la importancia de la estructura familiar en la nutrición de los niños/as.

Palabras clave: Estructura familiar, nutrición infantil, acortamiento.

Abstract

The role of family structure on stunting (low height-for-age) in Argentinian preschool children aged 2-5This study assesses the relationship between family structure and stunting among children aged 2-5 in Argentina. Data for this study was drawn from the first wave of the Argentinian National Nutrition and Health Survey 2005 ( $\mathrm{N}=11$ 632). Results from logistic regression models show that, although socioeconomic variables have a significant effect on stunting, family structure also matters. The odds of stunting among children living in extended nuclear families are lower than those of children living in strictly nuclear ones. Furthermore, children from single parent households do not have higher odds of stunting than those from two-parent families. Our work highlights the importance of family structure for child nutrition.

Key words: Family structure, child nutrition, stunting. 


\section{INTRODUCCIÓN}

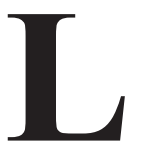

os cambios en los patrones familiares observados desde la segunda mitad del siglo pasado en los países de alto ingreso (Andersson, 2003; Bianchi y Casper, 2000) generaron un número considerable de investigaciones orientadas al estudio de las consecuencias que estos cambios podrían tener en el bienestar de menores de distintas edades. En general, estas investigaciones estaban, y aún están, orientadas a analizar el efecto que los cambios en la estructura familiar tienen sobre indicadores tales como nivel educativo alcanzado (Aquilino, 1996; Cavanagh et al., 2006), desarrollo de habilidades cognitivas (Carlson y Corcoran, 2001), bienestar social y emocional (Amato, 2005; Deleire y Kalil, 2002; Wen, 2008) y estado de salud, tanto física como mental, de los menores que viven en hogares no tradicionales (Blackwell, 2010; Bzostek y Beck, 2011; Bramlett y Blumberg, 2007), entendiéndose por hogar tradicional al constituido por una familia nuclear.

Las familias en América Latina y el Caribe, particularmente las urbanas, también se han ido diversificando, como ha ocurrido en los países de altos ingresos de América del Norte y Europa, de acuerdo a la etapa de la transición demográfica en la que se encuentran las distintas sociedades latinoamericanas. Según la Comisión Económica para América Latina y el Caribe, durante las últimas décadas se observa, en la región, un aumento considerable en el número de hogares unipersonales y de aquellos con jefatura femenina a la vez que el número de hogares biparentales y de familias nucleares muestra un notable descenso (CEPAL, 2004).

En los países de medianos y bajos ingresos, los estudios sobre la salud de los niños y niñas se centran fundamentalmente en su estado nutricional, especialmente en la presencia de desnutrición. ${ }^{1}$ La combinación entre desnutrición y enfermedades infecciones es todavía uno de los mayores problemas a los que se enfrenta la salud pública en estos países (Rice et al., 2000). La desnutrición incluye manifestaciones tales como bajo peso respecto de la edad, baja talla respecto de la edad (acortamiento), y extremada delgadez respecto de la talla (emaciación) (UNICEF, 2006).

La desnutrición es considerada como un factor de riesgo importante para el desarrollo de distintos tipos de enfermedades y otros resultados que no se limitan al ámbito de la salud (Grantham-McGregor et al., 2007). Por ejemplo, estudios para América Latina y el Caribe han mostrado que

\footnotetext{
${ }^{1}$ La desnutrición es definida como el resultado de una insuficiente ingesta alimentaria en concurrencia con el padecimiento de diferentes enfermedades infecciosas (UNICEF, 2006).
} 
el acortamiento y la emaciación están relacionados con la manifestación de emociones negativas (Flores Villavicencio et al., 2005) y que el acortamiento a edades tempranas está relacionado con déficits educacionales y cognitivos, tanto en la infancia (Freeman et al., 1980) como en la adolescencia (Walker et al., 2005). También se ha encontrado una relación entre acortamiento, obesidad y trastornos en el desarrollo motriz, social y emocional de los infantes (Bove et al., 2012).

Estudios realizados con datos tanto de África y Asia como de los Estados Unidos de América muestran evidencias adicionales sobre la relación existente entre estructura familiar y malnutrición en la infancia. Por ejemplo, se ha observado que en Etiopia el riesgo de acortamiento es mayor entre los menores viviendo en familias nucleares monoparentales, particularmente aquellas donde sólo la madre está presente, que entre aquellos menores que viven en familias nucleares completas o en familias extendidas (Gurmu y Etana, 2013). Asimismo, con datos recientes de las Encuestas de Demografía y Salud (DHS) para África ${ }^{2}$ y Asia $^{3}$ se ha observado que los menores viviendo en hogares monoparentales de madres divorciadas, o cuyas uniones se han disuelto, presentan un mayor riesgo de acortamiento que aquellos nacidos de una primera unión que continúa (DeRose et al., 2014). En contraste, utilizando también datos de la DHS 1986-1988 para países de África del Oeste, ${ }^{4}$ otros autores no encontraron ninguna relación significativa entre la estructura familiar (madre en matrimonio monógamo, en matrimonio polígamo, y no en unión) y la relación talla por edad de los infantes (Desai, 1992).

Si bien para América Latina y el Caribe los estudios que analizan el efecto de la estructura familiar sobre el estado nutricional de niños y niñas no abundan, se puede decir que, en mayor o menor medida, los resultados observados coinciden con aquellos que fueron señalados en el párrafo anterior para otras regiones del mundo. Por ejemplo, la observación de DeRose et al. (2014) para países de África y Asia sobre acortamiento en menores viviendo en familias monoparentales con madre presente se mantiene en países de América Central, Sudamérica, y el Caribe. ${ }^{5}$ Sin embargo, contrariamente a las observaciones para África del Oeste, Desai (1992)

\footnotetext{
${ }^{2}$ Camerún (2011), El Chad (2004), Etiopía (2011), Gana (2008), Kenia (2008-2009), Nigeria (2008), República Democrática del Congo (2007), Tanzania (2010) y Uganda (2011).

${ }^{3}$ Bangladés (2011), Filipinas (2008), India (2005-2006), Indonesia (2012), Pakistán (2006-2007) y Vietnam (2002).

${ }^{4}$ Ghana, Malí y Senegal.

${ }^{5}$ Bolivia (2008), Colombia (2010), Haití (2012), Honduras (2011-2012), Perú (2012), República Dominicana (2007).
} 
señala que para los países de América Latina y el Caribe analizados ${ }^{6}$ existe una relación entre el estado conyugal de las madres y la relación talla por edad; es decir, los infantes viviendo en hogares biparentales cuyos padres se encuentran en una unión consensual están expuestos a un mayor riesgo de acortamiento que aquellos cuyos padres están formalmente casados. Coincidentemente, Bronte-Tinkew y DeJong (2004), utilizando datos de la ronda 1996 para Jamaica del estudio Living Standard Measurement Study Surveys, muestran que los menores viviendo en familias nucleares cuyos padres están en una unión consensual o en familias monoparentales se encuentran expuestos a un riesgo mayor de acortamiento que aquellos que viven en familias nucleares cuyos padres están formalmente casados. Por otro lado, Fernald y Neufeld (2007), con datos para siete estados mexicanos como parte de la National Social Welfare Survey 2003 de México, encuentran que los menores que viven en familias en las que el padre está ausente están expuestos a un riesgo mayor de acortamiento que los otros. Es importante señalar que los estudios mencionados hasta ahora incluyen en sus análisis características demográficas y socioeconómicas a fin de permitir analizar el efecto independiente que tiene la estructura familiar en la desnutrición infantil.

En referencia a la Argentina, si bien existen estudios sobre el estado nutricional de niños y niñas (Bejarano et al., 2005; Bolzán et al., 2005; Cesani et al., 2013; Kovalskys et al., 2011), sus causas y su relación con el nivel cognitivo, el nivel educacional, la presión arterial, y la salud tanto visual como oral (Acosta et al., 2009; Calvo et al., 2005; Lacunza, 2010; Martínez y Lucas, 2004), hasta donde llega nuestro conocimiento sobre el tema, no se han producido estudios que analicen el efecto de la estructura familiar sobre el estado nutricional de preescolares.

Por lo tanto, el objetivo fundamental de este trabajo es explorar la influencia que tiene la estructura familiar en la salud de niños y niñas de dos a cinco años de edad, caracterizada por el estado nutricional de éstos, en Argentina. Para tal efecto se utiliza la Encuesta Nacional de Nutrición y Salud (ENNyS) en su ronda 2005. Esta es la primera encuesta en el país que posee datos antropométricos a nivel nacional y la única disponible hasta el momento. ${ }^{7}$ Con esta investigación esperamos contribuir a la literatura que explora la influencia de la familia en la salud y bienestar de los niños y niñas de América Latina y el Caribe.

\footnotetext{
${ }^{6}$ Brasil, Colombia y República Dominicana.

${ }^{7}$ La ENNyS 2005 es la primera encuesta nacional de salud y nutrición realizada en Argentina. Si bien una segunda ronda fue programada para 2015, a la fecha no hay datos disponibles.
} 
El marco conceptual subyacente en los estudios sobre el estado nutricional de niñas y niños fue construido por UNICEF (1998), al incorporar la desnutrición en el contexto amplio del desarrollo económico y social. Según esta perspectiva teórica, la desnutrición infantil y de la niñez es el resultado de un conjunto de factores multisectoriales que operan a nivel individual, de la familia y la sociedad. De modo que las causas de la malnutrición pueden clasificarse en factores inmediatos, subyacentes y básicos. Por lo tanto, no es posible estudiar la desnutrición sin tener en cuenta, por ejemplo, la influencia de factores socioeconómicos y culturales. Por consiguiente, este marco referencial proporciona un enfoque integral para identificar los riesgos asociados con los distintos factores. La familia puede ser entonces entendida como una unidad intermedia de análisis para la conexión entre dichos factores. La familia, tiene entonces un papel mediador entre el contexto socioeconómico y el niño o la niña. ${ }^{8}$

La organización de este documento es la siguiente: en la primera sección describimos en detalle la metodología utilizada para medir el estado nutricional de los niños y niñas; en la segunda, describimos la fuente de datos utilizada; en la tercera las variables y el método estadístico. Luego se presentan los resultados obtenidos y se discuten sus implicaciones. Finalmente, se presentan las conclusiones del estudio.

\section{Materiales y metodos}

\section{Determinación del estado nutricional de niños y niñas}

La recomendación internacional para establecer los niveles de malnutrición de una población y luego realizar una interpretación adecuada de los resultados es la consideración de las denominadas medidas antropométricas, como la talla y el peso en relación a la edad de los menores (de Onis y Blössner, 2003). Los indicadores más comunes son peso por edad, peso por talla, y talla por edad. La estimación de estos indicadores requiere la comparación con un grupo de referencia. Para tales efectos esta investigación utiliza el paquete estadístico WHO Anthro ${ }^{9}$ desarrollado por la Organización Mundial de la Salud (OMS). El paquete utiliza los estándares obtenidos por la OMS (WHO Child Growth Standards) basados en un estudio que involucró a niños y niñas saludables de diversos países (WHO Multicentre Growth Reference Study Group, 2006).

\footnotetext{
${ }^{8}$ Ver UNICEF (1998).

${ }^{9}$ WHO Anthro (versión 3.2.2, enero 2011) disponible en: http://www.who.int/childgrowth/software/en/
} 
Para la comparación con los valores estándar, el paquete WHO Anthro utiliza el estadístico Z-score (Puntaje Z) que se define como la desviación de un valor individual (observado) del valor medio de la población de referencia dividido por el valor de la desviación estándar (DS) de la población de referencia (de Onis y Blössner, 1997). Es decir, se utiliza el sistema Z-score para calcular el número de DS sobre o por debajo del valor medio de referencia de la medida antropométrica que se está considerando. Los puntos de corte o umbrales utilizados son los siguientes:

- Talla por edad: < - 2 DS - clasificará como baja talla respecto de la edad (acortamiento).

- Peso por talla: <-2 DS - clasificará como bajo peso respecto de la talla (emaciación) .

- Peso por edad: < - 2 DS - clasificará como bajo peso respecto de la edad (bajo peso).

\section{Fuente de datos}

Como se mencionó anteriormente, los datos utilizados en este estudio provienen de la ENNyS 2005 que fue elaborada por el Ministerio de Salud de la Nación Argentina. Uno de los objetivos de la ENNyS es la evaluación del estado de nutrición y salud de los habitantes del país, basándose en indicadores alimentarios, antropométricos y bioquímicos. La población objetivo de esta encuesta son los infantes de 6 a 23 meses, los niños y niñas de dos a cinco años de edad, las mujeres y embarazadas de 10 a 49 años de edad (Ministerio de Salud, 2007). Esta encuesta se basó en una muestra probabilística que cubrió todos los segmentos sociales de la población objetivo. Si bien la encuesta se realizó a nivel nacional, geográficamente sólo se consideró a la población urbana en localidades con cinco mil o más habitantes (de acuerdo al Censo Nacional de Población 2001).

La ENNyS sólo considera hogares particulares y los define de la siguiente manera: un hogar particular es "el constituido por toda persona o personas que comparten una misma vivienda bajo el régimen de tipo familiar y consumen alimentos con cargo al mismo presupuesto, independientemente de que sean parientes o no" (Ministerio de Salud, 2007: 22). Si bien hogar y familia no responden al mismo concepto, debido al modo en que la ENNyS define un hogar, en este trabajo los términos hogar y familia se utilizarán indistintamente dado que se excluyeron a niños y niñas que viven en hogares que incluyen personas sin relación de parentesco. El Jefe o Jefa del Hogar es aquella persona que sirve como punto de referencia para la 
determinación del arreglo familiar dentro del hogar a través de la relación de parentesco de cada individuo respecto de esta persona. Ciertos atributos personales del Jefe o Jefa de Hogar se utilizan habitualmente para deducir características del hogar en su conjunto. En el presente trabajo nos referiremos, en términos generales, al Jefe o Jefa del Hogar como la Persona de Referencia. Dado que la relación de parentesco de las personas dentro del hogar se obtiene en relación a la persona de referencia, sólo se puede identificar completamente la composición de la familia a la cual pertenece un menor cuando este es un hijo o hija de esta persona. Estos menores constituyen alrededor de 80 por ciento de los niños y niñas de dos a cinco años para los que la ENNyS provee algún tipo de información.

Para el presente trabajo, se han clasificado las familias analizadas en cuatro grupos, a saber:

1. Nuclear: está constituida por la Persona de Referencia, su pareja, uno o más hijos de dos a cinco años de edad e hijos de otras edades si estos vivieran en el hogar que se está considerando. Desafortunadamente ENNyS no diferencia entre parejas en unión consensual y parejas en unión formal.

2. Nuclear Extensa: es una familia de tipo Nuclear que incluye uno o más parientes.

3. Monoparental: está constituida por la Persona de Referencia, uno o más hijos de dos a cinco años de edad e hijos de otras edades si estos vivieran en el hogar que se está considerando.

4. Monoparental Extensa: es una familia del tipo Monoparental que incluye uno o más parientes.

En la ENNyS existen 12363 niños y niñas de entre dos y cinco años de edad que tienen una estructura familiar identificable y para los que se puede obtener el Z-score correspondiente a la relación talla por edad y/o a la relación peso por talla.

\section{Métodos estadísticos y descripción de variables}

Del análisis descriptivo de los datos se observa que en la población de niños y niñas de dos a cinco años de edad la prevalencia de acortamiento de estatura es 7.5 por ciento ( 925 casos), de bajo peso es 2.1 por ciento (261 casos) y de emaciación 1.2 por ciento (142 casos). Cabe señalar que tanto la prevalencia de acortamiento como la de bajo peso y la de emaciación son similares a las obtenidas para Argentina basadas en otra fuente de datos usando los mismos estándares de la OMS para el grupo etario bajo con- 
sideración (Padula et al., 2012). En América Latina, en general, ya se ha documentado una baja prevalencia de emaciación (Victora, 1992), similar a la encontrada en el presente estudio.

Debido a la reducida prevalencia de bajo peso y de emaciación, el presente estudio analiza el efecto de la estructura familiar en el estado nutricional de menores de dos a cinco años centrándose en el acortamiento de talla. La relación entre estructura familiar y acortamiento se modelará utilizando un modelo logístico.

Variables de Ajuste. Los modelos serán ajustados por Edad y Sexo de los menores, Región, Sexo de la Persona de Referencia, Tamaño del Hogar, Educación de la Persona de Referencia, Índice de Riqueza, y Asistencia Alimentaria.

Región: existen en Argentina siete regiones con características muy diversas. Por lo tanto, la variable Región es una variable categórica con siete categorías: Ciudad de Buenos Aires (CABA) que es la categoría de referencia, Gran Buenos Aires, Cuyo, Noreste (NEA), Noroeste (NOA), Pampeana, y Patagonia (Ministerio de Salud, 2007). En particular, la vasta área norte (conformada por las regiones NEA y NOA) que, si bien no es homogénea, presenta características socioeconómicas, demográficas y sanitarias que la diferencian del resto del país: elevado porcentaje de población rural, de niños y adolescentes, pobreza, analfabetismo, población sin cobertura médica y desocupación/subocupación (PNUD, 2005).

Tamaño del Hogar: la variable Tamaño del Hogar controla por la cantidad de personas en el hogar ajustada por la composición demográfica. Esto permite obtener lo que se conoce como adulto equivalente. Esta medida de equivalencia refleja las necesidades energéticas/calóricas específicas por edad y sexo, utilizando como unidad de referencia a un hombre adulto de 30 a 59 años de edad. A esta unidad se la denomina adulto equivalente y se le asigna un valor igual a uno (INDEC, 2012; Morales 1998). ${ }^{10}$ Se establecieron 3 categorías básicas para la variable Tamaño del Hogar: Pequeño (menos de tres adultos equivalentes), Mediano (entre tres y cinco adultos equivalentes), y Numeroso (más de cinco adultos equivalentes).

Educación de la Persona de Referencia: como se señaló con anterioridad, en muchos casos no se puede determinar quién es la madre del niño o niña que se está considerando. Por lo tanto, se tomó el nivel educacional de la persona de referencia, y no el nivel educacional de la madre del me-

\footnotetext{
${ }^{10}$ Por ejemplo, un hogar compuesto por un hombre de 35 años, una mujer de 30 años y un niño o niña de un año tiene un tamaño de 2.17. Este número se obtiene sumando las medidas de adulto equivalente de sus integrantes: 1 (hombre 30-59 años), 0.74 (mujer 18-59 años) y 0.43 (niño o niña de un año).
} 
nor, como una aproximación al nivel educacional de la persona que toma decisiones de consumo que pueden afectar al hogar en su conjunto. Se consideraron cuatro categorías: nivel primario incompleto, primario completo, nivel secundario incompleto, y secundario completo y más.

Índice de Riqueza: este índice es una medida resumen del bienestar económico de los hogares. El procedimiento seguido para su construcción es el propuesto por Filmer y Pritchett (2001) quienes mostraron que este tipo de índice es robusto y confiable para la estimación de la riqueza en el largo plazo. Las variables que se utilizaron para la construcción de este índice son: tipo de vivienda, material del piso, cantidad de personas por habitación (excluyendo cocina y baño), forma de abastecimiento de agua, tipo de servicio sanitario y si la vivienda cuenta con servicio de electricidad, refrigerador, y línea telefónica (terrestre). Se utilizó el método de componentes principales ${ }^{11}$ para asignar pesos a las distintas componentes de este índice. El promedio de los valores del Índice de Riqueza es 0 y su DS 1. A los efectos de facilitar la interpretación del Índice de Riqueza se lo re-escaló sumándole 5 unidades de modo tal que sus valores sean todos positivos. Para el análisis estadístico, se consideró la distribución por cuartiles del Índice de Riqueza, por lo que se definieron cuatro segmentos ${ }^{12}$. El primer segmento representa al grupo de hogares con "mayor bienestar económico" y el cuarto segmento al grupo de hogares con "menor bienestar económico."

La información para la construcción del Índice de Riqueza se obtuvo utilizando la muestra completa de niños y niñas de ENNyS (seis meses a cinco años de edad). Aunque la muestra sólo incluye viviendas en zonas urbanas, algunas de ellas están ubicadas en asentamientos precarios sin, o con mínimo, acceso a los servicios estándares de la ciudad. Por lo tanto, este indicador, aunque amplio, captura razonablemente bien la variación del bienestar económico de los hogares presentes en la muestra.

Asistencia Alimentaria: al momento de realizar el relevamiento de los datos existían en Argentina varios programas gubernamentales destinados a asistir con alimentos a poblaciones nutricionalmente vulnerables (niños, mujeres embarazadas, discapacitados, y adultos mayores). Estos planes gubernamentales proveen alimentos en especie, incluyendo leche, cupones o vales, y/o comidas en instalaciones comunitarias (Aulicino, 2012). Ade-

${ }^{11}$ El método de componentes principales es un algoritmo matemático utilizado para reducir la dimensionalidad de los datos, en este caso el número de las variables que se utilizaron para la construcción del índice, sin perder la variabilidad de los mismos.

${ }^{12}$ Estos segmentos no están perfectamente balanceados debido al elevado número de valores repetidos lo que ha dificultado la determinación de los puntos de corte. 
más de los programas financiados por el estado también existen programas respaldados por organizaciones no gubernamentales, religiosas y otras. Para reflejar el hecho de que el hogar recibe algún tipo de ayuda alimentaria se utiliza en el análisis una variable dicotómica con valor 1 si cualquier integrante del hogar recibió algún tipo de ayuda alimentaria en los últimos tres meses y 0 en caso contrario.

\section{Resultados}

\section{Análisis descriptivo}

De los 12363 niños y niñas de dos a cinco años de edad solamente en 11 632 casos se cuenta con información para todas las variables de interés. La Tabla 1 muestra la composición de la muestra analítica. Todos los menores en la muestra viven con uno o los dos padres en hogares que no incluyen personas que no están en relación de parentesco. La gran mayoría de los menores, 78.6 por ciento, viven en hogares nucleares (con hermanas o hermanos si es que hay alguno en el hogar); 8.4 por ciento vive en un hogar monoparental. En 13 por ciento de los casos las familias consideradas son nucleares extensas o monoparentales extensas. En estas familias los parientes son fundamentalmente abuelas y/o abuelos. De estos, solamente 10.4 por ciento vive en hogares nucleares extensos, 2.7 por ciento restante lo hace en un hogar monoparental extenso (Tabla 1).

De los hogares que incluyen algún familiar, sólo 20.5 por ciento son monoparentales. En general, en las familias monoparentales la persona de referencia es una mujer (87.8 por ciento de los casos). Lo contrario ocurre en las familias nucleares, en 89.2 por ciento de los casos la persona de referencia es hombre. Esto puede obedecer a razones culturales dado que habitualmente se designa como jefe del hogar a un hombre. El tamaño promedio de los hogares (en unidades de adulto equivalente) es 3.8. Del total de hogares, 38.9 por ciento recibe algún tipo de ayuda alimentaria.

Con respecto a las variables relacionadas con el nivel socioeconómico, podemos decir que, alrededor de 40 por ciento de los niños y niñas viven en hogares donde la persona de referencia tiene por lo menos educación secundaria completa y sólo diez por ciento vive en hogares donde esta persona no tiene educación primaria completa. El índice de riqueza captura importantes diferencias en el bienestar económico de los hogares, fundamentalmente entre el primer cuartil (el que indica el nivel de bienestar más elevado) y el cuarto (el que indica el nivel de bienestar más bajo). 
Tabla 1: Análisis descriptivo: Muestra analítica compuesta por 11632 niños y niñas de dos a cinco años de edad

\begin{tabular}{|c|c|c|c|c|c|c|}
\hline Variables & $\%$ & Media & $\mathrm{DE}$ & Mediana & Percentil 10 & Percentil 90 \\
\hline Edad en meses & & 43.0 & 10.7 & 43.2 & 28.0 & 57.7 \\
\hline Z-score edad-talla & & -0.5 & 1.1 & -0.5 & -1.8 & 0.9 \\
\hline \multicolumn{7}{|l|}{ Acortamiento } \\
\hline Sí & 7.6 & & & & & \\
\hline \multicolumn{7}{|l|}{ Sexo menores } \\
\hline Mujer & 49.9 & & & & & \\
\hline \multicolumn{7}{|l|}{ Región } \\
\hline CABA & 3.5 & & & & & \\
\hline Conurbano & 3.5 & & & & & \\
\hline Cuyo & 12.4 & & & & & \\
\hline NEA & 17.5 & & & & & \\
\hline NOA & 21.7 & & & & & \\
\hline Pampeana & 16.7 & & & & & \\
\hline Patagonia & 24.8 & & & & & \\
\hline \multicolumn{7}{|l|}{ Tipo de familia } \\
\hline Nuclear & 78.6 & & & & & \\
\hline Nuclear extensa & 10.4 & & & & & \\
\hline Monoparental & 8.4 & & & & & \\
\hline Monoparental extensa & 2.7 & & & & & \\
\hline \multicolumn{7}{|c|}{ Sexo persona de referencia } \\
\hline Mujer & 19.3 & & & & & \\
\hline Tamaño del hogar & & 3.8 & 1.5 & 3.4 & 2.3 & 5.7 \\
\hline Pequeño & 34.8 & 2.5 & 0.4 & 2.6 & 2.2 & 2.9 \\
\hline Mediano & 48.7 & 3.8 & 0.6 & 3.7 & 3.1 & 4.6 \\
\hline Numeroso & 16.5 & 6.4 & 1.3 & 6.0 & 5.2 & 8.1 \\
\hline \multicolumn{7}{|c|}{ Nivel educación persona de referencia } \\
\hline Primario incompleto & 10.0 & & & & & \\
\hline Primario completo & 26.2 & & & & & \\
\hline Secundario incompleto & 23.9 & & & & & \\
\hline $\begin{array}{l}\text { Secundario completo } \\
\text { y más }\end{array}$ & 39.8 & & & & & \\
\hline Índice de riqueza & & 5.0 & 1.0 & 5.3 & 3.4 & 5.8 \\
\hline Primer segmento & 21.3 & 5.9 & 0.2 & 5.8 & 5.7 & 6.2 \\
\hline Segundo segmento & 26.3 & 5.6 & 0.1 & 5.6 & 5.5 & 5.7 \\
\hline Tercer segmento & 25.6 & 5.1 & 0.2 & 5.1 & 4.7 & 5.3 \\
\hline Cuarto segmento & 26.9 & 3.5 & 0.9 & 3.7 & 2.3 & 4.4 \\
\hline $\begin{array}{l}\text { Ayuda alimentaria } \\
\text { Sí }\end{array}$ & 38.9 & & & & & \\
\hline
\end{tabular}

Nota: DE por desviación estándar.

Fuente: elaboración propia a partir de datos de la ENNyS 2005. 
La Tabla 2 muestra la distribución de la prevalencia de acortamiento de estatura de acuerdo a las variables socioeconómicas que se usan en el análisis multivariado. Si bien las familias monoparentales son las que presentan mayor prevalencia de acortamiento, la diferencia entre la prevalencia de acortamiento de los menores en este grupo familiar y aquellos en familias nucleares y nucleares extensas es estadísticamente diferente sólo a diez por ciento. Si tenemos en cuenta el sexo de la persona de referencia, la única diferencia que se encuentra es en las familias nucleares donde la mayor prevalencia de acortamiento se observa en aquellos hogares con jefe mujer, pero, como en el caso anterior, esta diferencia es significativa sólo a diez por ciento.

Como era de esperarse, la prevalencia de acortamiento es más pronunciada en las provincias que integran la región NEA. También, de acuerdo con lo esperado, se observa un claro gradiente creciente en la prevalencia de acortamiento según los hogares estén ubicados en los distintos segmentos del índice de riqueza (con la menor prevalencia en el primer segmento, el más beneficiado económicamente). Asimismo, según lo esperado, existe un gradiente claro si se toma en cuenta la educación de la persona de referencia (mayor prevalencia de acortamiento entre menores que viven en un hogar en el que la persona de referencia no completó sus estudios primarios).

Cuando se considera el tamaño del hogar se observa que la prevalencia de acortamiento aumenta con el aumento del tamaño del hogar, variando de alrededor de seis por ciento entre menores que viven en "hogares pequeños" a casi el doble entre aquellos que viven "hogares numerosos." Cabe destacar que, si bien la variable Tamaño del Hogar está basada en lo que se denomina adulto-equivalente, los hogares que responden a la definición de Hogar Pequeño tienen entre dos y cuatro integrantes en 99.4 por ciento de los casos, aquellos hogares definidos como Hogar Mediano tienen entre cuatro y seis integrantes en 94.7 por ciento de los casos, y los Hogares Numerosos entre siete y once integrantes en 91.3 por ciento de los casos.

Con respecto a la ayuda alimentaria, se observa acortamiento en cinco por ciento de los niños y niñas en hogares que no reciben ningún tipo de ayuda. Esta prevalencia se duplica entre aquellos que viven en hogares que reciben algún tipo de ayuda alimentaria. 
Tabla 2: Prevalencia de acortamiento en 11632 niños y niñas de dos a cinco años de edad según determinadas características

\begin{tabular}{|c|c|c|c|}
\hline Variables & Acortamiento \% & Variables & Acortamiento \% \\
\hline Sexo menores & & Tamaño del hogar & \\
\hline Mujer $(\mathrm{N}=5831)$ & 7.5 & Pequeño ( $\mathrm{N}=4$ 036) & 5.7 \\
\hline \multirow[t]{2}{*}{ Varón $(\mathrm{N}=5$ 801) } & 7.6 & Mediano $(\mathrm{N}=5671)$ & 7.0 \\
\hline & & Numeroso ( $\mathrm{N}=1925)$ & 13.0 \\
\hline Región & & Índice de riqueza & \\
\hline $\mathrm{CABA}(\mathrm{N}=408)$ & 6.1 & Primer Segmento $(\mathrm{N}=2476)$ & 3.9 \\
\hline Conurbano $(\mathrm{N}=406)$ & 7.4 & $\begin{array}{l}\text { Segundo Segmento }(N=3 \\
074)\end{array}$ & 5.1 \\
\hline Cuyo $(\mathrm{N}=1446)$ & 6.0 & Tercer Segmento (N = 2977$)$ & 7.2 \\
\hline NEA $(\mathrm{N}=2033)$ & 10.0 & Cuarto Segmento $(\mathrm{N}=3132)$ & 13.2 \\
\hline $\mathrm{NOA}(\mathrm{N}=2524)$ & 8.0 & & \\
\hline Pampeana (N = 1934) & 8.0 & & \\
\hline Patagonia $(\mathrm{N}=2881)$ & 6.4 & & \\
\hline Nivel educación persona de referencia & & Ayuda Alimentaria & \\
\hline Más que secundario completo $(\mathrm{N}=4631)$ & 4.8 & No $(\mathrm{N}=7$ 101) & 4.8 \\
\hline Secundario incompleto $(\mathrm{N}=2778)$ & 6.8 & Sí (N=4 531) & 11.9 \\
\hline Primario completo $(\mathrm{N}=3053)$ & 9.8 & & \\
\hline Primario incompleto $(\mathrm{N}=1170)$ & 14.6 & & \\
\hline \multicolumn{4}{|l|}{ Tipo de familia (según persona de referencia) } \\
\hline Nuclear (N = 9144$)$ & 7.4 & Monoparental (N=974) & 9.1 \\
\hline Hombre (N = 8209$)$ & 7.3 & Hombre $(\mathrm{N}=109)$ & 11.0 \\
\hline Mujer $(\mathrm{N}=935)$ & 8.8 & Mujer (N=77) & 8.9 \\
\hline Nuclear extensa $(\mathrm{N}=1204)$ & 7.0 & $\begin{array}{l}\text { Monoparental Extensa } \\
(\mathrm{N}=310)\end{array}$ & 8.1 \\
\hline Hombre $(\mathrm{N}=1025)$ & 6.7 & Hombre $(\mathrm{N}=47)$ & 6.4 \\
\hline Mujer $(\mathrm{N}=179)$ & 8.4 & Mujer $(\mathrm{N}=263)$ & 8.4 \\
\hline
\end{tabular}

Fuente: elaboración propia a partir de datos de la ENNyS 2005. 


\section{Análisis bivariado}

La Tabla 3 muestra los resultados obtenidos de la estimación de modelos bivariados de regresión logística en los que la variable dependiente es el logito de la probabilidad de que un niño o niña de dos a cinco años de edad presente acortamiento de estatura. ${ }^{13} \mathrm{E}$ análisis bivariado muestra que los coeficientes asociados a cada una de las variables mencionadas anteriormente, excepto sexo de los menores, son estadísticamente significativos. Vivir en la zona NEA, en una familia monoparental, en un hogar numeroso o mediano, recibir ayuda alimentaria, que la persona de referencia tenga un nivel educativo menor que el de educación secundaria completa, y que el nivel de bienestar económico del hogar sea bajo, como era de esperarse, incrementan las chances que tienen los menores de padecer acortamiento.

\section{Análisis multivariado}

La Tabla 4 muestra los resultados obtenidos de la estimación de tres modelos de regresión logística anidados. El primer modelo (Modelo 1), el más simple, sólo incluye las variables que indican la región del país donde vive el menor, su edad y sexo, así como la variable asociada a la estructura familiar. ${ }^{14} \mathrm{Al}$ igual que en el análisis bivariado, los resultados muestran que las chances de que un niño o niña que vive en la región NEA presente acortamiento son 70 por ciento mayores que para un niño o niña que vive en la CABA. La estimación del Modelo 1 también muestra que las chances de presentar acortamiento aumentan 25 por ciento si el niño o niña vive en un hogar monoparental, comparado con un hogar nuclear. Al igual que en el modelo bivariado, el sexo de los menores no está relacionado con las chances de presentar acortamiento. Sin embargo, las chances de presentar acortamiento disminuyen en uno por ciento por cada mes de incremento en la edad de los menores. El Modelo 2 incluye, además de las variables presentes en el Modelo 1, la variable que describe la cantidad de personas que viven en el hogar en términos de adulto-equivalente.

El Modelo 2 muestra que, al incorporar la variable relacionada con el tamaño del hogar, las chances de que un niño o niña presente acortamiento viviendo en cualquier región del país no son diferentes a las de un menor viviendo en la CABA.

\footnotetext{
${ }^{13}$ Debido a que en un hogar puede haber más de un niño o niña las regresiones ajustan por efecto de agrupamiento en el hogar, lo que se expresa en errores estándar (ES) robustos.

${ }^{14}$ Por las razones expuestas con anterioridad los términos familia y hogar en nuestro caso se utilizan en forma intercambiable.
} 
Tabla 3: Regresión logística bivariada - Variable dependiente: logito de la probabilidad de acortamiento en 11632 menores de 2 a 5 años de edad

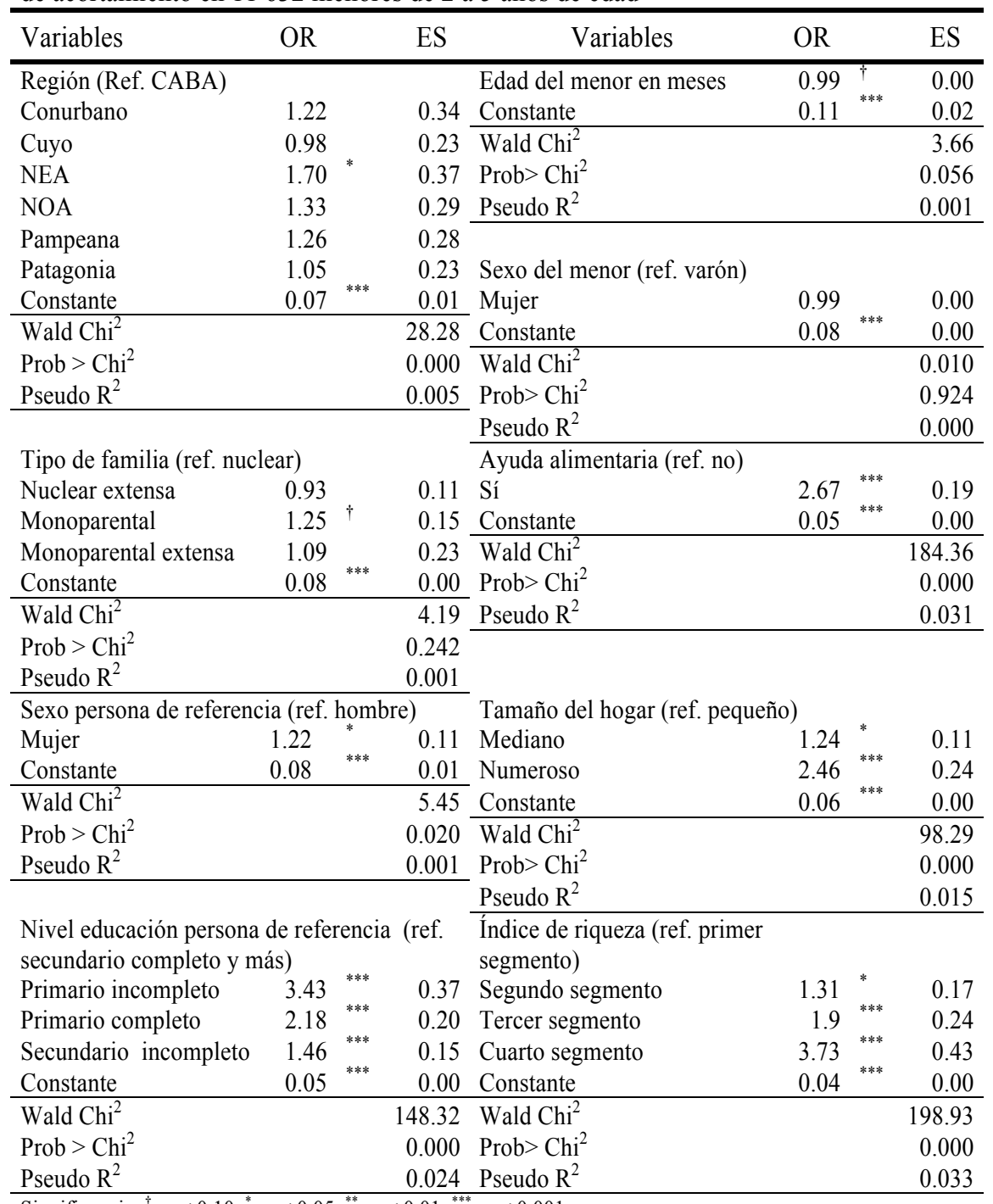

Significancia: ${ }^{\dagger}: \mathrm{p}<0.10,{ }^{*}: \mathrm{p}<0.05,{ }^{* *}: \mathrm{p}<0.01,{ }^{* * *}: \mathrm{p}<0.001$.

Nota: Los errores estándar (ES) de los 4 modelos están ajustados por agrupamiento en los hogares.

Fuente: elaboración propia a partir de datos de la ENNyS 2005. 
Tabla 4: Regresión logística multivariada - Variable dependiente: logito de la probabilidad de acortamiento en 11632 menores de 2 a 5 años de edad

\begin{tabular}{|c|c|c|c|c|c|c|c|c|}
\hline \multirow{2}{*}{ Variables } & \multicolumn{2}{|c|}{ Modelo 1} & \multicolumn{3}{|c|}{ Modelo 2} & \multicolumn{3}{|c|}{ Modelo 3} \\
\hline & OR & ES & OR & & ES & OR & & ES \\
\hline \multicolumn{9}{|l|}{ Región (Ref. CABA) } \\
\hline Conurbano & 1.22 & 0.34 & 1.12 & & 0.32 & 0.85 & & 0.25 \\
\hline Cuyo & 0.98 & 0.23 & 0.87 & & 0.21 & 0.77 & & 0.19 \\
\hline NEA & $1.69^{*}$ & 0.37 & 1.41 & & 0.31 & 0.93 & & 0.21 \\
\hline NOA & 1.33 & 0.29 & 1.12 & & 0.25 & 0.80 & & 0.18 \\
\hline Pampeana & 1.25 & 0.28 & 1.13 & & 0.26 & 0.90 & & 0.21 \\
\hline Patagonia & 1.04 & 0.23 & 0.95 & & 0.21 & 0.86 & & 0.20 \\
\hline Edad en meses & $0.99 \dagger$ & 0.00 & 0.99 & ** & 0.00 & 0.99 & $*$ & 0.00 \\
\hline \multicolumn{9}{|c|}{ Sexo menores (ref. varón) } \\
\hline Mujer & 0.99 & 0.07 & 1.00 & & 0.07 & 1.00 & & 0.07 \\
\hline \multicolumn{9}{|c|}{ Tipo de familia (ref. nuclear) } \\
\hline Nuclear extensa & 0.91 & 0.11 & 0.67 & ** & 0.08 & 0.75 & $*$ & 0.10 \\
\hline Monoparental & $1.25^{\dagger}$ & 0.15 & 1.35 & * & 0.17 & 1.10 & & 0.16 \\
\hline Monoparental extensa & 1.06 & 0.23 & 0.93 & & 0.20 & 0.90 & & 0.21 \\
\hline \multicolumn{9}{|c|}{ Tamaño del hogar (ref. pequeño) } \\
\hline Mediano & & & 1.36 & ${ }^{* *}$ & 0.12 & 1.17 & $\dagger$ & 0.11 \\
\hline Numeroso & & & 2.74 & $* * *$ & 0.28 & 1.80 & *** & 0.21 \\
\hline \multicolumn{9}{|c|}{ Sexo persona de referencia (ref. hombre) } \\
\hline Mujer & & & & & & 1.00 & & 0.11 \\
\hline \multicolumn{9}{|c|}{ Nivel educación persona de referencia (ref. secundario completo y más) } \\
\hline Primario incompleto & & & & & & 1.54 & *** & 0.19 \\
\hline Primario completo & & & & & & 1.23 & * & 0.13 \\
\hline Secundario incompleto & & & & & & 1.04 & & 0.11 \\
\hline \multicolumn{9}{|c|}{ Índice de riqueza (ref. primer segmento) } \\
\hline Segundo segmento & & & & & & 1.07 & & 0.14 \\
\hline Tercer segmento & & & & & & 1.21 & & 0.17 \\
\hline Cuarto segmento & & & & & & 1.88 & ${ }^{* * *}$ & 0.26 \\
\hline \multicolumn{9}{|c|}{ Ayuda Alimentaria (Ref. No) } \\
\hline Sí & & & & & & 1.72 & *** & 0.15 \\
\hline Constante & $0.09^{* * *}$ & 0.02 & 0.08 & *** & 0.02 & 0.08 & ${ }^{* * *}$ & 0.02 \\
\hline Df & & 12 & & & 14 & & & 22 \\
\hline AIC & & 6218 & & & 6122 & & & 5942 \\
\hline $\mathrm{BIC}$ & & 6306 & & & 6225 & & & 6104 \\
\hline
\end{tabular}

Significancia: ${ }^{\dagger}: \mathrm{p}<0.10,{ }^{*}: \mathrm{p}<0.05,{ }^{* *}: \mathrm{p}<0.01,{ }^{* \pi * *}: \mathrm{p}<0.001$.

Nota: Los errores estándar de los 4 modelos están ajustados por agrupamiento en los hogares. Df: por grados de libertad Fuente: elaboración propia a partir de datos de la ENNyS 2005. 
Los resultados del modelo 2 indican que las chances de presentar acortamiento de un menor viviendo en un hogar monoparental, comparadas con la de un menor en un hogar nuclear, aumentan en diez por ciento respecto del modelo anterior. Además, la estimación del Modelo 2 muestra que las chances de presentar acortamiento disminuyen en cerca de 30 por ciento si el niño o niña vive en un hogar nuclear extenso comparado con un hogar nuclear. Ahora bien, el efecto beneficioso de vivir en un hogar nuclear extenso se compensa con el efecto negativo de vivir en un hogar de tamaño medio y se elimina si el hogar es numeroso (las chances de acortamiento de un niño o niña viviendo en un hogar numeroso son casi tres veces más que las que tendría viviendo en un hogar de tamaño pequeño).

El Modelo 3, que es el mejor ajusta a los datos, agrega al Modelo 2 variables relacionadas con el nivel socioeconómico del hogar (Sexo de la Persona de Referencia, Tamaño del Hogar, Educación de la Persona de Referencia, Índice de Riqueza, y Asistencia Alimentaria). Lo primero que se destaca en la estimación del Modelo 3 es el efecto adverso de vivir en un hogar monoparental. No sólo el valor del coeficiente asociado al hogar monoparental es menor que en el modelo anterior, sino que ya no es estadísticamente significativo. El efecto favorable de vivir en un hogar nuclear extenso no se ve afectado.

Por otro lado, como era de esperarse, las chances de acortamiento aumentan si el niño o niño vive en un hogar en el que la persona de referencia sólo tiene educación a nivel primario ( 54 por ciento si no completó este nivel y 23 por ciento si lo completó) comparado con aquellos en los que la persona de referencia tiene, por lo menos, educación a nivel secundario completo. Igualmente, las chances de acortamiento para un niño o niña son mayores si vive en un hogar que pertenece al nivel inferior del índice de riqueza ( 88.0 por ciento si el hogar pertenece al cuarto segmento) comparadas con las chances de acortamiento de aquellos cuyo hogar está en el primer segmento (los más beneficiados económicamente).

La ayuda alimentaria (dada por el gobierno u otras instituciones), es un indicador válido del estatus socioeconómico. Por lo tanto, como se esperaba, las chances de acortamiento de los niños y niñas que viven en hogares que reciben este tipo de ayuda son mayores (72 por ciento) que las de aquellos que no la reciben.

\section{DisCUSIÓN}

El objetivo principal de este estudio es investigar rol que tiene la estructura familiar en el acortamiento de la estatura en preescolares argentinos. 
Nuestros resultados muestran que la relación entre estructura familiar y las chances de acortamiento de un niño o niña de dos a cinco años de edad está mediada por la posición económica de la familia. En particular, nuestro análisis indica que el pertenecer a una familia monoparental aumenta las chances de acortamiento con respecto a las de un niño en una familia nuclear a través del efecto de la situación económica familiar. Por otro lado, y contrariamente a lo esperado, las chances de presentar acortamiento son menores entre los niños y niñas que viven en una familia nuclear extensa, comparadas con las de aquellos en una familia nuclear.

Este último resultado es consistente con los resultados obtenidos por Crookston y colegas $(2010)^{15}$ quienes mostraron que, no sólo el acortamiento es menos severo entre los infantes de 6 a 18 meses de edad que viven en hogares con abuelos residentes, que para aquellos que viven en otro tipo de hogar, sino también que las chances de alcanzar la talla adecuada entre los cuatro y seis años de edad que tenían los infantes que anteriormente presentaban acortamiento era mayor si estos tenían abuelos en el hogar. Nuestros resultados para los niños o niñas en familias monoparentales extensas muestran un efecto protector del acortamiento similar al de las familias nucleares extensas, aunque en este caso el coeficiente asociado a este tipo de familias no es estadísticamente significativo (la muestra analítica contiene sólo 310 casos de este tipo de familias, 2.7 por ciento). Cabe señalar, que en la muestra que se está analizando en el presente estudio en más de 80 por ciento de las familias extensas, ya sea nucleares o monoparentales, los parientes que residen en el hogar son abuelas o abuelos.

También se podría afirmar que nuestros resultados son consistentes con el obtenido por Schmeer (2013) ${ }^{16}$ quien estudió anemia (otro indicador de desnutrición) en niños y niñas mexicanos de 3 a 12 años de edad. Schmeer encontró que la co-residencia con los abuelos maternos tiene un efecto beneficioso, independientemente del tipo de arreglo familiar.

Aubel (2012) también resalta el rol fundamental que cumplen las abuelas en la vida familiar y comunitaria en sociedades no occidentales de Asia, África y América Latina. Ellas aconsejan y educan a las mujeres jóvenes en todos los aspectos que hacen al bienestar del hogar e influyen sobre la toma de decisiones de los padres y otros hombres de la familia (Aubel, 2012).

Cuando se incluye en el Modelo 2 la interacción entre tipo de familia y sexo de la persona de referencia (resultados no mostrados), se observa que

${ }^{15}$ El estudio de Crookston et al. (2010) fue realizado sobre 1674 niños y niñas peruanos del Young Lives Study.

${ }^{16}$ Este estudio está basado en una muestra de 4649 niños y niñas del Mexican Family Life Survey. 
para la categoría monoparental con jefatura femenina las chances de acortamiento son mayores (y estadísticamente significativas a diez por ciento) que las de un de un niño o niña en un hogar nuclear. El coeficiente asociado a esta categoría pierde significancia estadística en el Modelo 3 con la inclusión de las variables que ajustan por estatus socioeconómico. Lo que sugiere, como hemos mencionado anteriormente, que para un niño o niña las desventajas nutricionales de vivir en un hogar monoparental con jefatura femenina estarían mediadas por factores relacionados con el bienestar económico del hogar.

Diversos estudios han señalado la mayor prevalencia de acortamiento que se observa en las provincias argentinas de las regiones NEA y NOA (Acosta et al., 1993; Bolzán et al., 2005; Bolzán y Mercer, 2009). Nuestros resultados muestran que una vez consideradas las variables relacionadas con el hogar (estructura familiar, jefatura, y tamaño ajustado por las necesidades energéticas de sus integrantes según edad y sexo), las chances de acortamiento de un niño o niña de cualquier región del país no son estadísticamente diferentes a las chances que tiene un menor viviendo en la Ciudad de Buenos Aires. Ahora bien, cabe destacar que los hogares definidos como "numerosos" se concentran principalmente en las provincias de las regiones NEA y NOA (23.4 y 27.9 por ciento, respectivamente).

En conformidad con la literatura existente, nuestros resultados muestran la influencia favorable que tiene una mayor educación de la persona de referencia en las chances de acortamiento de los menores (Desai, 1992; Frost et al., 2005): las chances de acortamiento son mayores para menores viviendo en hogares en los que la persona de referencia no completó, o sólo completó, el nivel de educación primario comparadas con las de un menor en un hogar en que la persona de referencia ha completado, como mínimo, el nivel secundario. El otro indicador de posición socioeconómica, el Índice de Riqueza, también muestra resultados que están en conformidad con la literatura disponible sobre el tema. Las chances de acortamiento aumentan entre los niños y niñas que viven en hogares menos beneficiados económicamente (Castro et al., 2005).

Un resultado más difícil de interpretar es el obtenido en relación a la ayuda alimentaria. Por un lado, debemos recordar que no siendo el presente un análisis longitudinal no podemos establecer causalidad. Por el otro, el signo del coeficiente asociado a la variable Ayuda Alimentaria estaría en sentido contrario al intuitivamente esperado, es decir, que el hecho de que algún integrante del hogar reciba ayuda alimentaria de algún tipo resulte en detrimento del estado nutricional de los menores. Nuestra interpretación es 
que dicha variable refleja las necesidades del hogar que recibe la ayuda y de acuerdo a esto el resultado obtenido es similar al de las otras variables que reflejan estatus socioeconómico.

Diversos estudios sobre los determinantes de la desnutrición de niños y niñas muestran resultados contradictorios respecto de las diferencias que existen entre sexos sobre las chances de acortamiento. Por ejemplo, mientras que Castro y sus colegas (2005) encontraron una mayor chance de acortamiento para las niñas que para los niños ${ }^{17}$ Martorell y Young (2012) encontraron lo contrario. ${ }^{18}$ En nuestro estudio no hemos encontrado diferencias estadísticamente significativas en las chances de acortamiento entre niños y niñas.

Un elemento muy importante de nuestro estudio es el tamaño de la muestra que se utilizó, compuesta por más de 11 mil niños y niñas de entre dos a cinco años de edad. A pesar del considerable tamaño de esta muestra, el estudio tiene algunas limitaciones. Primero, la limitación característica de los factores no observados que pueden seleccionar a los niños y niñas a pertenecer a algún tipo particular de familia y que pueden estar relacionados con la desnutrición. Segundo, siendo un estudio de corte transversal no podemos inferir relaciones de causalidad sino simplemente de asociación. Tercero, las categorías familia nuclear y familia monoparental incluyen diferentes grupos que, dadas las características de la ENNyS, no son posibles de identificar y que pueden implicar distintos tipos de actitud en lo referente a la crianza y la alimentación de los hijos. Por ejemplo, los padres en las familias nucleares pueden estar casados formalmente o cohabitando y el padre o la madre en las familias monoparentales pueden diferir entre ellos por ser solteros o divorciados. Cuarto, nuestra muestra no incluye hogares cuyo Jefe o Jefa no sean el padre o madre del menor en cuestión, ni tampoco hogares que incluyan personas que no son parientes. Por lo tanto, nuestros resultados no se pueden generalizar a este tipo de familias y la muestra termina siendo no representativa a nivel nacional.

\section{Conclusiones}

Si bien las características socioeconómicas tienen un efecto significativo sobre el acortamiento de la estatura de los preescolares argentinos entre dos y cinco años de edad, nuestro análisis identifica el rol independiente que tiene la estructura familiar en los niveles de desnutrición de este grupo

${ }^{17}$ Castro et al. (2005) estudiaron 3240 menores de 11 años en el departamento de Antioquia en Colombia.

${ }^{18}$ Martorel y Young (2012) analizaron una muestra de 10317 menores de cinco años en Guatemala (Reproductive Health Survey) 
poblacional. Los resultados indican que los preescolares en familias nucleares extensas, que incluyen en su gran mayoría a uno o ambos abuelos, tienen menores chances de acortamiento que aquellos en familias estrictamente nucleares. También encontramos que la relación entre pertenecer a una familia monoparental y las chances de acortamiento está mediada por la posición económica de la familia.

El estudio del acortamiento en la infancia es importante en términos de intervenciones sociales futuras, dado que se han identificado efectos a largo plazo en diversas áreas como el desarrollo cognitivo, el nivel educacional alcanzado, y la productividad económica en la edad adulta (Dewey y Begum, 2011).

\section{REFERENCIAS BIBLIOGRÁFICAS}

Acosta, L.D., Maffrand, R.A., Alonso, I. Aspitia, M., Farina, F., Gaite, E., Peñalba, T., Sanchez, O., Segura, F., Sirabo, C., Estario, J. C. y Fernández, A.R., 2009, "Evaluación de la salud visual y el estado nutricional en escolares de una localidad de la provincia de Córdoba. Importancia de su valoración en atención primaria de salud", en Revista de Salud Pública, 13(1): 60-68.

Andersson, G., 2003, Dissolution of unions in Europe: a comparative overview, en Max Planck Institute of Demographic Research Working Paper WP 2003-004, Rostock.

Amato, P.R., 2005, "The impact of family formation change on the cognitive, social, and emotional well-being of the next generation", in The Future of Children, 15(2): 75-96.

Aquilino, W.S., 1996, "The life course of children born to unmarried mothers: childhood living arrangements and young adult outcomes", in Journal of Marriage \& Family, 58: 293-310.

Aubel, J., 2012, "The role and influence of grandmothers on child nutrition: Culturally designated advisors and caregivers", in Maternal \& Child Nutrition, 8(1): $19-35$.

Aulicino, C., 2012, Una primera aproximación a las políticas de Educación Nutricional en las provincias argentinas. Documento de Trabajo $\mathrm{N}^{\circ}$ 90, mayo, Buenos Aires: Centro de Implementación de Políticas Públicas para la Equidad y el Crecimiento (CIPPEC).

Bejarano, I., Dipierri, J., Alfaro, E., Quispe, Y. y Cabrera, G., 2005, “Evolución de la prevalencia de sobrepeso, obesidad y desnutrición en escolares de San Salvador de Jujuy”, en Archivos Argentinos de Pediatría, 103(2): 101-109. 
Bianchi, S.M. y Casper, L.M., 2000, «American families», en Population Bulletin. Blackwell, D.L., 2010,"Family structure and children's health in the United States: findings from the National Health Interview Survey, 2001-2007", in Vital Health Statistics, Washington, 10(246).

Bolzán, A. y Mercer, R., 2009, "Seguridad alimentaria y retardo crónico del crecimiento en niños pobres del norte argentino", en Archivos Argentinos de Pediatría, 107(3): 221-228.

Bolzán, A., Mercer, R., Ruiz, V., Brawerman, J., Marx, J., Adrogué, G., Carioli, N. y Cordero, C., 2005, "Evaluación nutricional antropométrica de la niñez pobre del norte argentino: Proyecto encuNa", en Archivos Argentinos de Pediatría, 103 (6): 545-555.

Bove, I., Miranda, T., Campoy, C., Uauy, R. y Napol, M., 2012, "Stunting, overweight, and child development impairment go hand in hand as key problems of early infancy: Uruguayan case", in Early Human Development, 88(9):747-751.

Bramlett, M.D. y Blumberg, S.J., 2007, "Family structure and children's physical and mental health", in Health Affairs, 26(2): 549-558.

Bronte-Tinkew, J. y DeJong, G., 2004, “Children's nutrition in Jamaica: do household structure and household economic resources matter?", in Social Science and Medicine, 58(3): 499-514.

Bzostek, S.H. y Beck, A.N., 2011, "Familial instability and young children's physical health", in Social Science \& Medicine, 73: 282-292.

Calvo, E.B. y Aguirre, P., 2005, "Crisis de la seguridad alimentaria en la Argentina y estado nutricional en una población vulnerable", Coordinadores Provinciales de la Encuesta Antropométrica: Buenos Aires, en Archivos Argentinos de Pediatría, 103(1): 77-90.

Carlson, M.J., Corcoran, M.E., 2001, "Family structure and children's behavioral and cognitive outcomes", in Journal of Marriage and Family, 63: 779-792.

Castro, B.A., González, E.R., Alvarez, M.C y Segura, A.M., 2005, "Factors associated with stunted growth in children below 11 years of age in Antioquia, Colombia, 2004", in Colombia Médica, 42(2): 207-214.

Cavanagh, S.E., Schiller, K.S. y Riegle-Crumb, C., 2006, "Marital transitions, parenting, and schooling: exploring the link between family-structure history and adolescents' academic status", in Sociology of Education, 79(4): 329-354.

Cesani, M.F., Garraza, M., Bergel Sanchís, M.A., Torres, M.F., Quintero, F.A. y Oyhenart, E.E., 2013, "A comparative study on nutritional status and body composition of urban and rural schoolchildren from Brandsen District (Argentina)", in Plos One, 8(1), e52792.

CEPAL, 2004, "Estructuras familiares, trabajo doméstico y bienestar en América Latina", en Panorama Social de América Latina 2004, Comisión Económica para América Latina y el Caribe. Santiago de Chile. 
Crookston, B.T., Penny, M.E, Alder, S.C, Dickerson, T.T., Merrill, R.M., Stanford, J.B., Porucznik, C.A. y Dearden, K.A., 2010, "Children who recover from early stunting and children who are not stunted demonstrate similar levels of cognition", in Journal of Nutrition, 140: 1996-2001.

Deleire, T. y Kalil, A., 2002, "Good things come in threes: single-parent multigenerational family structure and adolescent adjustment", en Demography, 39(2): 393-413.

De Onis, M. y Blössner, M., 1997, WHO Global Database on Child Growth and Malnutrition, WHO/NUT/97.4. Organización Mundial de la Salud. Ginebra.

De Onis, M. y Blössner, M., 2003, “The World Health Organizaton Global Database on Child's Growth and Malnutrition: methodology and applications", in International Journal of Epidemiology, 32: 518-526.

DeRose, L., Corcuera, P., Gas, M., Molinero Fernández, L.C., Salazar, A. y Tarud, C., 2014, "Family instability and early childhood health in the devoloping world", in World Family Map 2014: Mapping Family Change and Child WellBeing Outcomes, An International Report from Child Trends.

Desai, S., 1992, "Children at risk: The role of family structure in Latin America and West Africa", in Population and Development Review, 18(4): 689-717.

Dewey, K.G. y Begum, K., 2011, "Long-term consequences of stunting in early life", in Maternal and Child Nutrition, 7(Suppl. 3): 5-18.

Fernald, L.C. y Neufeld, L.M., 2007, “Overweight with concurrent stunting in very young children from rural Mexico: prevalence and associated factors", in European Journal of Clinical Nutrition, 61: 623-632.

Filmer, D. and Pritchett, L.H., 2001, "Estimating wealth effects without expenditure data or tears: an application to educational enrollments in states of India", in Demography, 38(1): 115-132.

Flores Villavicencio, M. E., Troyo Sanromán, R., Haro Jiménez, L.P., Lemus García, A., Valle Barbosa, M.A. y Muñoz de la Torre, A., 2005, "La desnutrición del siglo XXI y su impacto en las emociones y afrontamientos en niños escolares", en Investigación en Salud, 7(3): 188-195.

Freeman, H., Klein, R.E., Townsend, J.W. y Lechtig, A., 1980, "Nutrition and cognitive development among rural Guatemalan children", in American Journal of Public Health, 70: 1277-1285.

Frost, M.B., Forste, R. y Haas, D., 2005, "Maternal education and child nutritional status in Bolivia: finding the links", in Social Science \& Medicine, 60: 395-407.

Grantham-McGregor, S., Cheung, Y.B., Cueto, S., Glewwe, P., Richter, L., Strupp, B., 2007, "Developmental potential in the first 5 years for children in developing countries", International Child Development Steering Group, in The Lancet, 369: 60-70.

Gurmu, E. y Etana, D., 2013, "Household structure and children's nutritional status in Ethiopia”, in Genus, 69(2): 113-130. 
INDEC, 2012, Canasta básica alimentaria y canasta básica total: historia, forma de cálculo e interpretación. Instituto Nacional de Estadística y Censos. Buenos Aires, Argentina. Disponible en http://www.indec.gov.ar/nuevaweb/cuadros/novedades/informe_canastas_basicas.pdf

Kovalskys, I., Rausch Herscovici, C. y De Gregorio, M.J., 2011, "Nutritional status of schoolchilren of Buenos Aires, Argentina: data using three references", in Journal of Public Health, 33(3): 403-411.

Lacunza, A.B., 2010, "Procesamiento cognitivo y deficit nutricional de niños en contexto de pobreza", en Psicología y Salud, 20(1): 77-88.

Martínez, S.E. y Lucas, G.Q., 2004, Correlación entre el estado nutricional y la condición bucal de los niños que concurren a la Cátedra de Odontopediatría de la FOUNNE, Universidad Nacional del Nordeste. Comunicaciones Científicas y Tecnológicas. Resumen M-053.

Martorell, R. y Young, M.F., 2012, "Patterns of stunting and wasting: potential explanatory factors" in American Society of Nutrition, 3: 227-233.

Ministerio de Salud, 2007, ENNyS. Encuesta de Nutrición y Salud. Documento de Resultados 2007, Ministerio de Salud. Presidencia de laNación. Disponible en http://datos.dinami.gov.ar/produccion/nutricion/material/A1a.pdf

Morales, E., 1988, Canasta básica de alimentos - Gran Buenos Aires, Documento de Trabajo 3, Buenos Aires: INDEC/IPA.

Padula, G., Seoane, A.I. y Salceda, S.A., 2012, "Variations in estimates of underweight, stunting, wasting, overweight and obesity in children from Argentina comparing three growth charts", in Public Health Nutrition 15 (11): 2086-2090.

PNUD, 2005, Informe de Desarrollo Humano Argentina 2005. Programa de Desarrollo de las Naciones Unidas, Buenos Aires.

Rice, A.L., Sacco, L., Hyder, A. y Black, R.W., 2000, “ Malnutrition as an underlying cause of childhooddeaths associated with infectious diseases in developing countries", in Bulletin of the World Health Organization, 78(10): 1207-1221.

Schmeer, K.K., 2013, "Family structure and child anemia in Mexico", in Social Science and Medicine, 95: 16-23.

UNICEF, 1998, The state of the world's chidlren 1998, Oxford University Press. Nueva York.

UNICEF, 2006, Progress for children: a report card for nutrition, Nueva York.

Victora, C.G., 1992, "The association between wasting and stunting: an international perspective", in Community and International Perspectives, 122: 1105-1110.

Walker, S.P., Chang, S.M., Powell, C.A. y Grantham-McGregor, S.M., 2005, "Effects of early childhood psychological stimulation and nutritional supplementaton on cognition and education in growth-stunted Jamaican children: prospective cohort study", in Lancet, 366: 1804-1807. 
Wen, M., 2008, "Family structure and children's health and behavior: data from the 1999 National Survey of America's Families", in Journal of Family Issues, 29(11): 1492-1519.

World Health Organization Multicentre Growth Reference Study Group, 2006, "WHO Child Growth Standards based on length/height, weight and age", en Acta Pcediatrica, 2006 (Suppl 450): 76-85.

\section{RESUMEN CURRICULAR DE LAS AUTORAS}

\section{Beatriz Novak}

Profesora Investigadora en el Centro de Estudios Demográficos, Urbanos y Ambientales, El Colegio de México. Doctora en Sociología y Maestra en Sociología por la Universidad de Wisconsin-Madison, Licenciada en Ciencias de la Computación por la Universidad de Buenos Aires. Entre sus más recientes publicaciones se encuentran (con coautor) "The enduring effects of smoking in Latin America" (American Journal of Public Health, 2015) y "Adult obesity, disease and longevity in Mexico" (Salud Pública de México, 2015).

Dirección electrónica: bnovak@colmex.mx

\section{Maria E. Muniagurria}

Profesora Asociada y Directora de la carrera de grado en el Departamento de Economía de la Universidad de Wisconsin-Madison (USA). Es Doctora en Economía por la Universidad de Minnesota (USA) y Licenciada en Economía por la Universidad Nacional de Buenos Aires (Argentina). Entre sus publicaciones recientes (con co-autores) destaca "Family Structure and Child Health in Argentina" (International Journal of Sociology of the Family, 2014).

Dirección electrónica: munia@ssc.wisc.edu

Artículo recibido el 2 de septiembre de 2015 y aprobado el 18 de febrero de 2017 . 